\title{
Burkitt's Lymphoma-A Case Report
}

\author{
Tarang C Arora ${ }^{1}$, Deval Arora ${ }^{2}$, Abhay M Tripathi ${ }^{3}$, Sonali Saha ${ }^{4}$, Kavita Dhinsa $^{5}$
}

\begin{abstract}
Introduction: Lymphomas are malignant neoplasms arising from lymphocytes B cell or T cell that affects mainly lymph nodes, spleen, and other nonhematopoietic tissues. They are classified as Hodgkin's lymphoma (HL) and non-Hodgkin's lymphoma (NHL). NHL is diagnosed in extranodal sites in $40 \%$ of cases, mainly in the head and the neck region, while HL is found in extranodal sites (1-4\%) with a lower incidence. Though it is poorly differentiated, rare, and aggressive, but it is known to have the shortest doubling time creating special challenges for diagnosis and treatment. In the oral soft tissues, lesions can occur as hard and diffuse tumors involving oral vestibule, gums, and posterior region of the hard palate. The extent of disease at presentation is the most important prognostic factor in Burkitt's lymphoma.

Management: Management modality includes chemotherapy as the mainstay of treatment for this disease; consultation with a hematologist and an oncologist should be obtained as soon as possible.

Aim: The following case report depicts a rare case of a 9-year-old male child with an aggressive form of Burkitt-like (BL) lymphoma highlighting the diagnosis, dental management, precautions, and general and dental considerations to be kept in mind in such special patients with immunocompromised status and considerations in such immunocompromised patients.
\end{abstract}

Keywords: Burkitt's lymphoma, Chemotherapy, Lymphoma, Non-Hodgkin's.

Journal of South Asian Association of Pediatric Dentistry (2019): 10.5005/jp-journals-10077-3017

\section{INTRODUCTION}

Lymphoma is a diverse malignant disease of the lymphatic system, with distinguished the presence of proliferated lymphoid cells or their precursors. It can be divided into two large groups: $\mathrm{HL}$ and $\mathrm{NHL}$ according to their aggressive behavior. ${ }^{1}$

NHL is commonly classified into three categories: (1) B-cell (NHL including Burkitt's and Burkitt-like (BL) lymphoma, and diffuse large B-cell lymphoma), (2) lymphoblastic lymphoma (primarily precursor T-cell lymphoma and less frequently precursor B-cell lymphoma), and (3) anaplastic large cell lymphoma (T-cell or nul cell lymphoma). ${ }^{2}$

$\mathrm{BL}$ is a high-grade B-cell neoplasm under the umbrella of NHL which is solid tumor of lymphoid organs. NHLs are generally clonal malignancies of the multiple cellular components of the normal lymph node, spleen, and thymus. ${ }^{2,3}$ Denis Burkitt, a British surgeon, working in the central Africa in Kampala, was the first to describe Burkitt's lymphoma in 1956. He noted facial swellings involving both upper and lower jaws, sometimes accompanied by proptosis in African children. They also had huge abdominal masses associated with facial swelling. Burkitt's lymphoma has also been found to be associated with an Epstein-Barr virus (EBV) and was proposed to be oncogenic. $^{3}$

It occurs predominantly in the first decades of life, mostly in males, and with significant affinity for the gnathic bones, especially the maxilla. The tumor usually progresses rapidly in the mouth and presents as a facial swelling or an exophytic mass that involves bones of the maxilla. It is typically characterized by dysregulation of the C-myc oncogene. ${ }^{2,4}$ The endemic form typically involves the mandible, maxilla, and abdomen. BL of the orofacial region typically occurs in the jaw bones associated with tooth mobility, dental pain, and jaw expansion. Sudden and unexpected development of life-threatening complications such as airway and abdominal obstruction and acute renal failure has been reported with $\mathrm{BL}$. In contrast, the sporadic form commonly
${ }^{1,3-5}$ Department of Pedodontics and Preventive Dentistry, Sardar Patel Post Graduate Institute of Dental and Medical Sciences, Lucknow, Uttar Pradesh, India

${ }^{2}$ Department of Dentistry, Autonomous State Medical College, Shahjahanpur, Uttar Pradesh, India

Corresponding Author: Deval Arora, Department of Dentistry, Autonomous State Medical College, Shahjahanpur, Uttar Pradesh, India, Phone: +91 9415315427, e-mail: devalarora@gmail.com

How to cite this article: Arora TC, Arora D, et al. Burkitt's Lymphoma-A Case Report. J South Asian Assoc Pediatr Dent 2019;2(1):25-28.

Source of support: Nil

Conflict of interest: None

presents as an abdominal mass involving the mesenteric lymph nodes or ileocecal region. ${ }^{5}$

Histologically, Burkitt's tumors are composed of monomorphic, medium-sized cells with round nuclei, multiple nucleoli, and relatively abundant basophilic cytoplasm, which may give the cells a "cohesive appearance." These tumors have an extremely high rate of proliferation, in addition to a high rate of cell death (apoptosis). A "starry sky" pattern is usually present, imparted by numerous benign macrophages that have ingested apoptotic tumor cells.

$B L$ occurs as an Epstein-Barr virus (EBV)-associated malignancy among children in the malaria belt of equatorial Africa (endemic $B L$ ), and sporadically in other geographical areas, where it also occurs among adults (sporadic BL).

$B L$ has also been frequently reported as a common neoplasm in HIV-infected patients, although it is not known why BL is so common in HIV and not in other forms of immunodepression. Thus, the term BL-like lymphoma has been commonly applied to those tumors that have morphological features intermediate between large cell lymphoma with centroblastic or immunoblastic features and typical BL. ${ }^{6}$ 


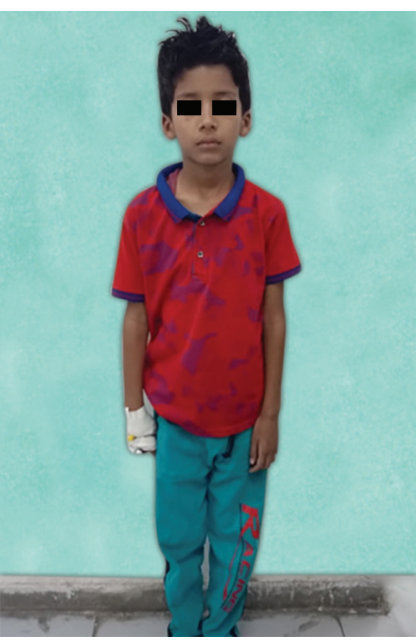

Fig. 1: Preoperative photograph

\section{Case Description}

A 9-year-old male patient reported at the department with the chief complaint of pain in the lower left back tooth region of the jaw (Fig. 1). Medical history revealed that the child was diagnosed with a mass in the terminal ileum which was confirmed as high-grade B-cell NHL at a prominent tertiary care center in the city. Cervical lymph nodes were palpable and pathological reports stated short segmental homogeneous mildly enhancing asymmetrical wall thickening with associated large soft tissue mass $(7.2 \times 4.2 \mathrm{~cm})$ involving a distal ileal loop causing partial luminal narrowing with mild proximal dilatation $(2.9 \mathrm{~cm})$ of the ileal loop as well. Initiation of chemotherapy treatment for the lymphoma was planned at a prominent tertiary care center in Lucknow city from where the patient was referred to the Department of Pedodontics and Preventive Dentistry for dental evaluation prior to initiation of chemotherapy for the treatment of lymphoma.

Clinical evaluation revealed the presence of two root stumps in relation to 74 and a treatment plan was formulated wherein removal of the same was planned before chemotherapy to avoid further infection. Orthopantomogram (OPG) was done to view the status of erupting succedaneous permanent teeth as the patient stated delayed eruption of lateral incisors since the age of the patient was 9 years. Additionally, it helped to view the position and eruption status of succedaneous permanent teeth and the root length and shape of root stump (74) to be extracted (Fig. 2). Routine blood investigations were done to assess the bleeding and coagulation status which shows satisfactory results. Referral was sent to a pediatrician and an oncologist to examine and give consent keeping in mind the current disease status evaluating the general health of the patient to undergo an invasive procedure like extraction.

Extraction of root stumps irt 74 was planned as soon as the patient reported back with the relevant documents after routine checkup. Critical attention was given to asepsis wherein all instruments were properly autoclaved before the use at $121^{\circ} \mathrm{C}$ for at least 30 minutes by using saturated steam under at least 15 psi of pressure. Before starting the procedure, the patient was kept under antibiotic prophylaxis for infection control (cap amoxicillin $250 \mathrm{mg}$ TDS $\times 7$ days) and povidone iodine (Betadine) was applied in the peripheral area around the oral cavity to maintain proper sterile work field. Inferior alveolar nerve block was administered to provide analgesia during the extraction procedure. Finally, extractions of root stumps were done using Straight Warwick James Elevator and mandibular root forceps (Fig. 3) and root
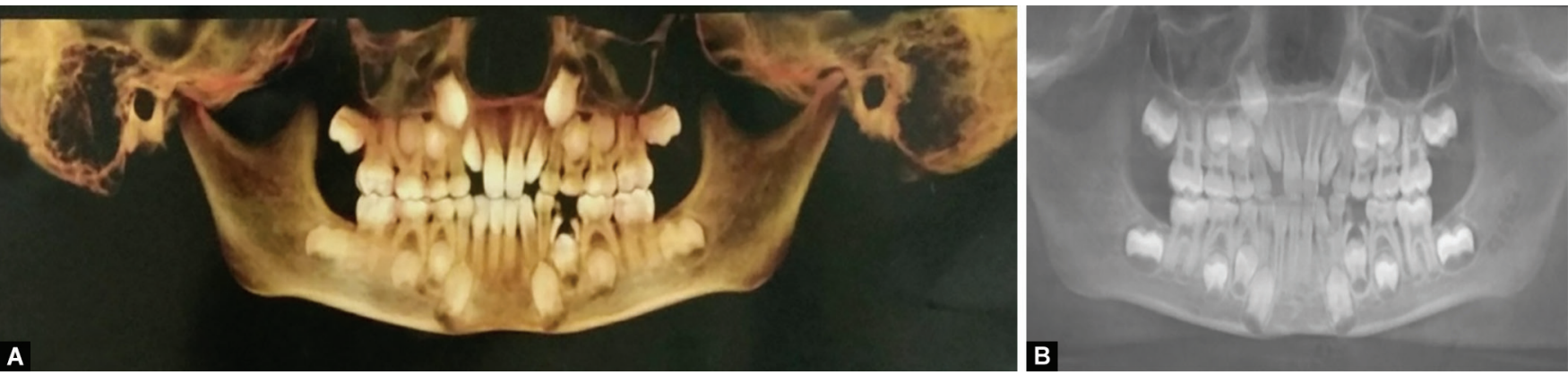

Figs 2A and B: (A) 2D-Cone beam computed tomography; (B) Orthopantomograph
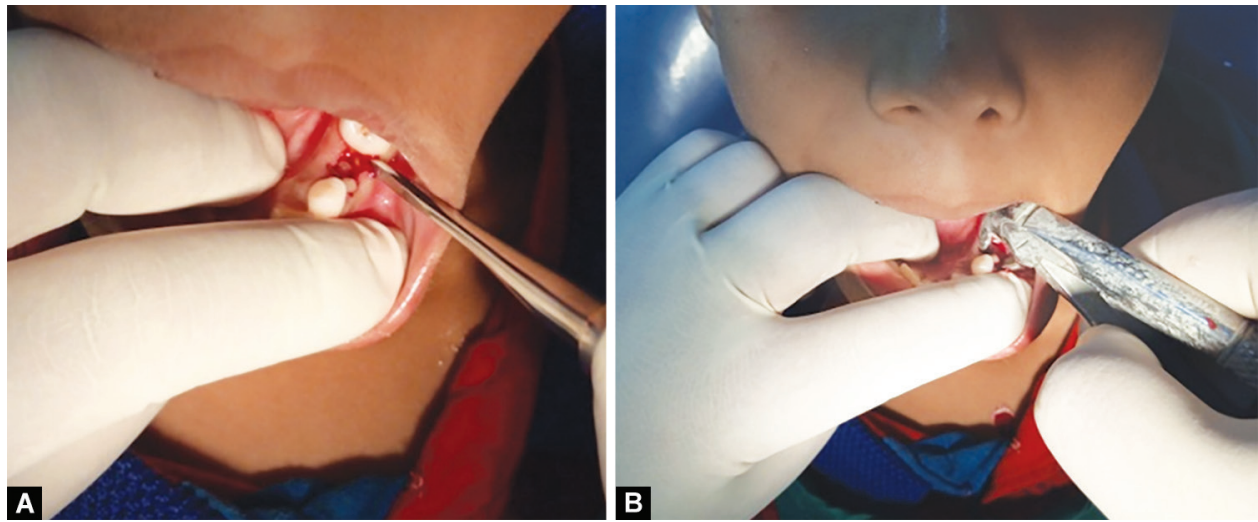

Figs $3 \mathrm{~A}$ and B: Removal of root stump irt 74 using a straight Warwick James elevator and mandibular root forceps. Preoperative photographs showing removal of root stump irt 74 


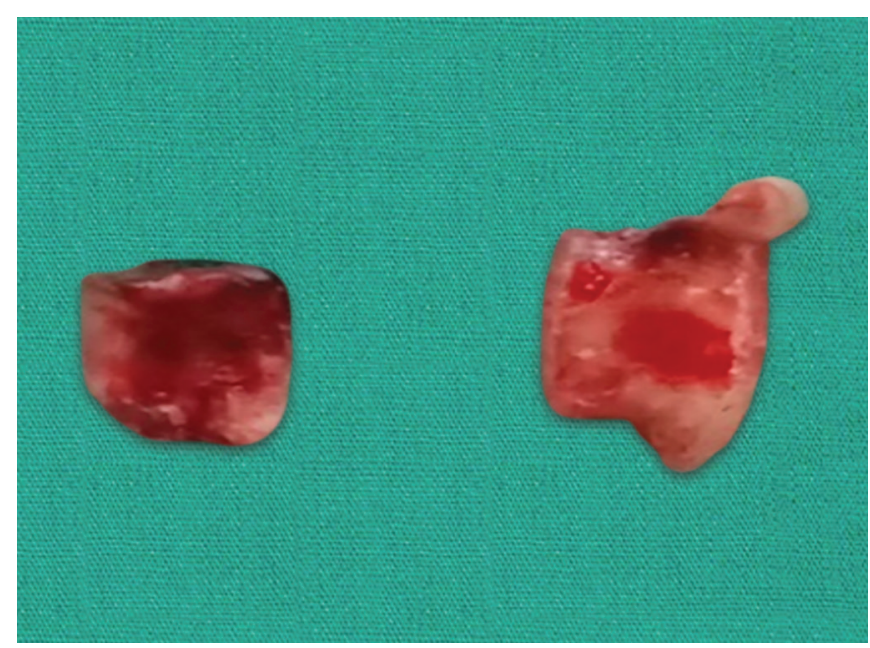

Fig. 4: Extracted root stumps irt 74

stumps irt 74 were removed atraumatically (Figs 4 and 5) followed by thorough irrigation of the socket with concentrated povidone iodine solution.

This was followed by giving a patient a pressure pack irt 74 to arrest bleeding. The extraction procedure was uneventful and postoperative instructions were given to the patient. The patient was finally discharged with antibiotic coverage for the next 7 days to promote healing. The patient was recalled next day to observe for any signs of postextraction complications such as pain, bleeding/increased inflammation, swelling, or infection. Nothing was reported by the patient and he was recalled after

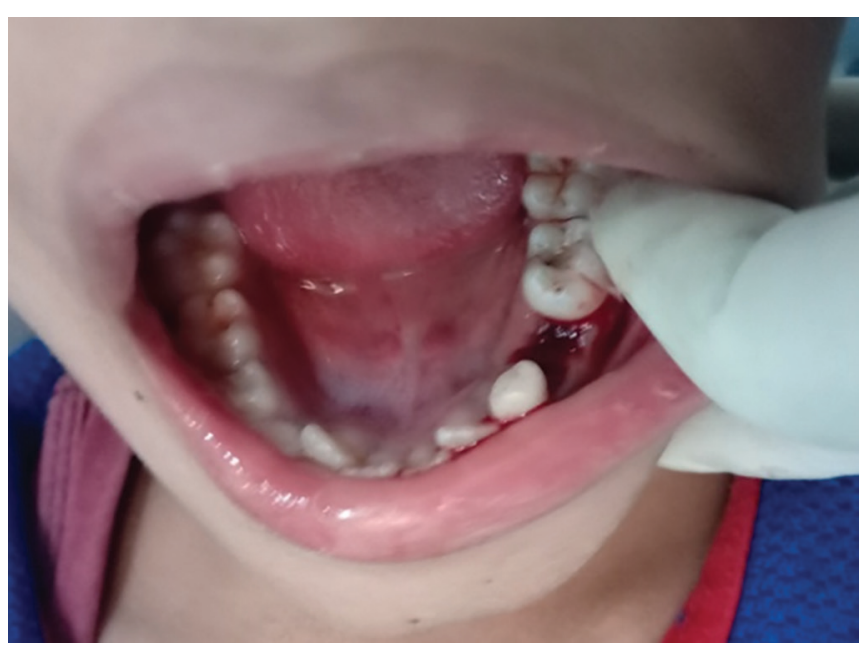

Fig. 5: Postoperative photograph

1 week follow-up, the healing was satisfactory with no evidence of any infection.

\section{Discussion}

NHLs are a group of diverse malignancies, usually involving lymph nodes. NLH is uncommon in an oral cavity and affects either soft tissue or bone. ${ }^{1}$

Dental management of immunocompromised patients has been a great challenge for a pediatric dentist. In the present case, a patient had NHL in the ileac region. In this respect, the purpose of dental evaluation prior to chemotherapy was to identify existing or

Table 1: Dental treatment before, during, and after chemotherapy ${ }^{10}$

\begin{tabular}{|c|c|c|}
\hline Before chemotherapy & During chemotherapy & After chemotherapy \\
\hline $\begin{array}{l}\text { The dentist should consult the oncologist to } \\
\text { determine the current condition of the patient } \\
\text { and the type of treatment planned }\end{array}$ & $\begin{array}{l}\text { The oncologist should be consulted to } \\
\text { know the degree of immune suppression } \\
\text { of the patient }\end{array}$ & $\begin{array}{l}\text { - The dentist should consult the } \\
\text { oncologist to determine immune } \\
\text { competence }\end{array}$ \\
\hline $\begin{array}{l}\text { Exhaustive examination of the oral cavity: discard } \\
\text { periapical lesions and/or bone alterations, and } \\
\text { the evaluation of periodontal health }\end{array}$ & $\begin{array}{l}\text { - Treatment of the complications of } \\
\text { chemotherapy (mucositis, xerostomia, etc.) }\end{array}$ & $\begin{array}{l}\text { - Insist on the need for routine } \\
\text { systematic oral hygiene }\end{array}$ \\
\hline $\begin{array}{l}\text { Denture fitting should be checked, with } \\
\text { readjustment or removal of those prostheses }\end{array}$ & & $\begin{array}{l}\text { - Use of chlorhexidine rinses and } \\
\text { fluorization }\end{array}$ \\
\hline
\end{tabular}
that prove traumatic

- Radiological study: intraoral (periapical and bitewing) and panoramic.

- General prophylactic measures: tartar removal, dental fluorization, and rinses with $0.12 \%$ chlorhexidine

- The patient should be informed of the complications of treatment

- Continued patient reminder of the need to maintain strict dental hygiene is indicated, with the added use of chlorhexidine rinses and fluorization

- Analgesics: paracetamol/metamizol

- No NSAID

- Antibiotics: dose adjustment is required according to the observed creatinine clearance values in patients with kidney problems

- Teeth that are non-viable or present a poor prognosis should be removed:

- Minor surgery: at least 2 weeks before chemotherapy

No elective dental treatment should be carried out

- Major surgery: 4-6 weeks before chemotherapy

Only emergency dental care 
potential oral infection and treat the same so that it does not hamper healing time. ${ }^{7}$ It is of utmost importance to evaluate the medical and hematological status of the patient before undergoing planned dental treatment. A platelet level of $100,000 / \mathrm{mm}^{3}$ is adequate for most of the dental procedure. ${ }^{8}$ Thus, a prior consent and all the necessary routine tests were conducted for the patient. Elective treatment procedures are deferred in such cases, majorly immediate extractions of decayed primary teeth are preferred under antibiotic cover.

The main oral complications and adverse sequelae of chemotherapy relate to conditions such as necrotic teeth, preexisting intrabony pathology, and advanced periodontal disease. Generalized periodontal diseases may produce lifethreatening infections and pyrexia in patients as they undergo chemotherapy. Such infections may necessitate an interruption of chemotherapy treatment, and, therefore, compromise the outcome of carcinoma treatment. ${ }^{7}$ Candidiasis is common in children with immunocompromised conditions; therefore, antifungal regime may be prescribed for the same. For reducing pain and inflammation while carrying dental procedures, nonsteroidal anti-inflammatory drugs (NSAIDs) and salicylates (drugs, altering the platelet function) must be avoided in thrombocytopenic patients. ${ }^{9}$

\section{Recommendations and Precautions}

For patients who are undergoing chemotherapy and necessitate extractions, gentle tissue management, appropriate suturing, and local hemorrhage control are indicated. Prior to extractions, the coagulation and bleeding parameters of the patient should be assessed (Table 1).

In this manner, patient's ability to tolerate chemotherapy may be greatly enhanced. Furthermore, excellent oral health has been consistently shown to improve general comfort of chemotherapy patients, resulting in fewer interruptions of chemotherapy treatment. ${ }^{11}$

\section{CONCLUSION}

Pediatric dentists must be aware of common hematological and oncological conditions and influence of these diseases on child's oral health. They should also have adequate knowledge of influence of chemotherapy and various drugs being prescribed to these children on oral health as well as various dental procedures and minor surgeries. It is important to take detailed history, general health status, hematological status, etc. before planning a dental procedure in such children. The postoperative care, instructions, and monitoring until satisfactory healing are a must before the patient is cleared and sent back to the physician/pediatrician/ oncologist for further management. The dental team was successful in managing an uneventful minor surgery in a child with Burkitt's lymphoma by following a systematic approach.

\section{References}

1. Silva TDB, Ferreira CBT, et al. Oral manifestations of lymphoma: a systematic review. Ecancermedicalscience 2016;10:665. DOI: 10.3332/ ecancer.2016.665.

2. Ghoroubi J, Mirshemirani A, et al. Abdominal Burkitt's lymphoma in children. Iran J Pediatr 2015;1(1):28-33.

3. Olaniyi JA. Burkitt Lymphoma: A Review. Journal of Open Access Scientific Reports 2012;1(6):2-4.

4. Freitas RDA, Veras Barros SSL, et al. Oral Burkitt's Lymphoma - Case Report. Rev Bras Otorrinolaringol 2008;74(3):458-461. DOI: 10.1590/ S0034-72992008000300023.

5. Patankar $S$, Venkatraman $P$, et al. Burkitt's lymphoma of maxillary gingiva: a case report. World J Clin Cases 2015;3(12):1011-1016. DOI: 10.12998/wjcc.v3.i12.1011.

6. Bellan C, Lazzi S, et al. Burkitt's lymphoma: new insights into molecular pathogenesis. J Clin Pathol 2003;56:188-193. DOI: 10.1136/ jcp.56.3.188.

7. Varun BR, Varghese NO, et al. Extranodal Non-Hodgkin's Lymphoma of the Oral Cavity: A Case Report. J Iran Med Sci 2017;42(4):407-411.

8. Fillmore, WJ, Leavitt, BD, etal. Dental extraction in thethrombocytopenic patient is safe and complications are easily managed. J Oral Maxillofac Surg 2013;71(10):1647-1652. DOI: 10.1016/j.joms.2013.05.011.

9. Risser A, Donovan D, et al. NSAID prescribing precautions. Am Fam Physician 2009;80(12):1371-1378.

10. Chaveli López B, Gavaldá Esteve C, et al. Dental treatment considerations in the chemotherapy patient. J Clin and Exp Dent 2011;3(1):e31-e42.

11. Moret Y, Perez C, et al. Non-Hodgkin's Lymphoma of Large Non-Cleft Cells of the Oral Cavity. Acta Odontol Venez 1999;37(3):1-15. 